\title{
An Innovative Approach to Development of Social Abilities in Individuals with Autism: A Pilot Study
}

\author{
Monica Mazza ${ }^{1^{*}}$, Maria Chiara Pino ${ }^{2}$, Melania Mariano $^{1}$ and Marco Valenti ${ }^{1,3}$ \\ ${ }^{1}$ Department of Applied Clinical Sciences and Biotechnology, University of L'Aquila, Italy \\ ${ }^{2}$ Department of Life, Health and Environmental Sciences, University of L'Aquila, L'Aquila, Italy \\ ${ }^{3}$ Reference Regional Centre for Autism, Abruzzo Region Health System, L'Aquila, Italy
}

"Corresponding author: Mazza M, Department of Applied Clinical Sciences and Biotechnology, University of L'Aquila, Via Vetoio, Località Coppito, 67100, L'Aquila, Italy, Tel: +39 0862 404141; E-mail: monica.mazza@cc.univaq.it

Rec date: January 29, 2016; Acc date: February 19, 2016; Pub date: February 26, 2016

Copyright: (c) 2016 Mazza M, et al. This is an open-access article distributed under the terms of the Creative Commons Attribution License, which permits unrestricted use, distribution, and reproduction in any medium, provided the original author and source are credited.

\begin{abstract}
Recent evidence suggests that individuals with Autism Spectrum Disorders (ASD) show a significant impairment in social abilities. There are no treatments that have demonstrated evidence of being effective at improving social skills and promoting independence. The transition to adulthood, which often involves loss of school support and child and adolescent mental health services, is a challenge in the rehabilitation field.

Our pilot study is the first that describes a novel approach to involving young adults with ASD in metacognitive exercise focused on social cognition. In treatment proposed, participants with ASD help each other to create software aimed at improving children with ASD's understanding of emotions. Our results showed an qualitative and quantitative improvement of their cognitive empathic abilities, as witnessed by parents and of the post- treatment assessment.
\end{abstract}

This pilot study highlights the importance to help individuals with ASD to fulfil their potential in areas of strength.

Keywords Autism spectrum disorders (ASD); Empathy; Rehabilitation intervention; Social competences

\section{Introduction}

Autism spectrum disorder (ASD) is a common neurodevelopmental syndrome with a strong genetic basis, characterised by qualitative impairments in social interaction / communication impairments and stereotypic behaviours [1-3]. Social cognition deficits seem to be "core" characteristics of ASD [4,5]. This social construct complex includes the cognitive processes for decoding and encoding the social world, such as Theory of Mind (ToM) and empathy [6]. ToM refers to the ability to attribute mental states to self and others, including knowledge, beliefs and intentions [7]. Other researchers have also included understanding feelings within the definition of ToM [5,8-10]. It is likely that ToM is a multidimensional process, requiring the integration of two components: cognitive and affective [8]. Cognitive ToM refers to the ability to make inferences about beliefs and motivations, while affective ToM refers to the ability to infer what a person is feeling. According to Shamay-Tsoory's model, cognitive ToM is a prerequisite for affective ToM, which also requires intact empathy processing. These abilities are essential in everyday life as they allows us to predict other people's behaviour and adjust own behaviour adequately $[8,11]$. Children with ASD have a restricted range of social skills, such as difficulties initiating interactions, maintaining reciprocity, giving and receiving social bids and responding to others to engage in successful interactions [12-15]. The most effective interventions for individuals with autism so far have been behavioural and educational [4]. The evidence for effectiveness of training in the social cognition precursors, such as joint attention, pretend play, socially synchronous behaviour, imitation, emotion recognition, theory of mind and functional communication were not established, but could be potentially effective [4]. Lai et al. stressed that the main goals of rehabilitative treatment for people with ASD are to maximise an individual's functional independence and quality of life through development and learning, improvements in social skills and communication, reductions in disability and comorbidity, promotion of independence and provision of support to families. Moreover, individuals should be helped with fulfilling their potential in areas of strength [4].

Several types of social skills interventions have been documented to improve social competencies and performance of school-age children with ASD [16,17]. Peer mediated intervention (PMI) approaches are considered evidence-based by the National Professional Development Center on ASD (http://autismpdc.fpg.unc.edu/content/ebp-update) and the National Standards Project (http:// www.nationalautismcenter.org) to improve social and communication deficits for children with ASD. More recently, it has been found that there are added benefits when this approach is combined with direct teaching of functional social communication skills in natural school contexts [15]. Little is known about the effectiveness of the treatment for adolescents and adults with ASD. Transition to adulthood, which often involves the loss of school support and child and adolescent mental health services, is an important challenge [4]. The end of secondary education is accompanied by slowed improvement, probably due to reduced occupational stimulation and insufficient adult services $[18,19]$. As far as we know, our pilot study is the first work in which four young adults with ASD help each other to create software aimed at improving children with ASD's understanding of emotions. Their work has a dual purpose: 
1. To create software to improve the recognition of emotions for children with ASD, created by adolescents and adults with the same syndrome

2. At same time, document the work of four young adult with ASD that represents a rehabilitative treatment that allows them to confront oneself with other person and improve their own social competencies. The innovative characteristic of treatment proposed is describing an approach that involved young adults with ASD in metacognitive exercises focused on social cognition abilities.

\section{Method}

\section{Participants}

The Reference Regional Centre for Autism of Abruzzo, San Salvatore Hospital, and L'Aquila, Italy recruited four young adults, ranging in age from 19 to 31 years. The ASD diagnosis was given by experienced clinicians (licensed psychiatrist and two psychologists) according to the new criteria of the DSM-5 [3]. All participants received a diagnosis of high-functioning autism made with Autism Diagnostic Observation Schedule Second Edition-ADOS-2 (Table 1) [20]. All had average to above-average estimated IQ score ranges according to the WAIS-III (Wechsler Adult Intelligence Scale, Third Edition) [21].

\begin{tabular}{|c|c|c|c|c|}
\hline & Subject 1 & Subject 2 & Subject 3 & Subject 4 \\
\hline Age (years) & 22 & 19 & 20 & 31 \\
\hline Education in years & 13 & 13 & 13 & 13 \\
\hline Gender & Male & Male & Male & Male \\
\hline ADOS-2 Total Score & 9 & 8 & 16 & 14 \\
\hline PIQ & 96 & 113 & 118 & 119 \\
\hline VIQ & 80 & 71 & 94 & 95 \\
\hline IQ Total & 85 & 86 & 109 & 104 \\
\hline
\end{tabular}

Table 1: Socio-demographic and clinical data of participants to study.

\section{Social cognition measures}

Advanced theory of mind task: This task is an Italian adaptation of a cognitive task used by Blair and Cipolotti and proposed in the literature by Happé $[22,23]$. The task consists of a short version of 13 vignettes, each accompanied by two questions-the comprehension question "Was it true what X said?" and the justification question "Why did X say that?" The 13 story types included Lie, White Lie, Joke, Pretend, Misunderstanding, Double Bluff, and Contrary Emotion. Each subject obtained a score ranging from 0 to 1 for each question. The maximum score was 13 .

Basic empathy scale-BES [24-26]: The BES is comprised of a total of 20 items. The participants had to give their ratings on a 5-point Likerttype scale $(1=$ Strongly Disagree, $2=$ Disagree, $3=$ Neither Agree nor Disagree, $4=$ Agree, $5=$ Strongly Agree). In the two-factor model, nine items assessed cognitive empathy (Items 3, 6, 9, 10, 12, 14, 16, 19, 20), and 11 items assessed affective empathy (Items 1, 2, 4, 5, 7, 8, 11, 13, $15,17,18)$. The BES demonstrated good validity $[25,26]$.
The Eyes Task is a revised version of the "Reading the Mind in the Eyes Test" [27]. In brief, participants were given 36 photographs depicting the ocular area in an equal number of different actors and actresses. At each corner of every photo, four emotional descriptors (e.g. dispirited, bored, playful or comforting) are printed, only one of which (the target word) correctly identifies the depicted person's mental state, while the others are included as foils. The test is scored by totalling the number of items (photographs) correctly identified by the participant; therefore, the maximum total score is 36 .

Emotion attribution task [22]: This task assessed the ability to represent the emotions of others. In this task, the participant was presented with 58 short stories describing an emotional situation and was required to provide an emotion describing how the main character might feel in that situation. The sentences were designed to elicit attributions of positive and negative emotions. This task assesses ability to identify and represent seven emotions: happiness, anger, disgust, sadness, embarrassment, envy and fearfulness. The task was scored according to the number of correct attributions.

The participants were assessed before and after treatment on social cognition measures.

\section{Treatment}

The treatment proposed aims to improve social cognition processes, such as empathy and theory of mind, in individuals with autism. The rehabilitation programme consists of a two-day-per-week treatment over a 24 -week period, with each session lasting approximately 60 minutes. The interesting aspect of this intervention is that participants are the creators and beneficiaries of the treatment at same time.

Each of the sessions included (1) the introduction of the topic (for example, the ability to discern others' emotions based on knowledge about social cues, such as gaze) and (2) a free discussion concerning the present topic. During discussions, the operator was encouraged to share experiences and feelings with others. (3) Subsequently, the young adults worked to create a rehabilitative programme for children with autism to help them understand the intentions and emotions of other people. This step is very important because participants are involved on metacognitive exercises focused on emotional and social abilities.

The four young adults used FaceGen software to create avatar faces. FaceGen is a face-generating 3D modelling middleware produced by Singular Inversions. A large number of different possible faces, either at random or from photographs, were selected. Advantages of its use (compared to using natural photos) is that a large number of stimuli can be generated easily, while the faces can be either matched or varied systematically according to characteristics such as race, gender, caricature, eye gaze, and head position.

The main objectives of our intervention were to:

1. Improve the awareness of one's emotional and mental states;

2. Improve the ability of empathic involvement in others' emotional experience;

3. Improve the capacity for adaptive coping with aversive or distressing emotions by using self-regulatory strategies [28].

4. Increase awareness that the structure of relationships is largely defined by how emotions are communicated within the relationship [28].

5. Increase the capacity for emotional self-efficacy [28]. 
Citation: Mazza M, Pino MC, Mariano M, Valenti M (2016) An Innovative Approach to Development of Social Abilities in Individuals with Autism:

6. Create a rehabilitative intervention from individuals with autism to other individuals with autism.

\section{Results}

The tests administered during the pre-intervention phase were compared with normative data (global cut-off) of a sample matched for age, sex and education (Table 2).

\begin{tabular}{|c|c|c|c|c|c|}
\hline & Subject 1 & Subject 2 & Subject 3 & Subject 4 & Cut-off \\
\hline Eyes Task & 10 & 20 & 23 & 26 & 26.2 (d.s.: $3.6 ;[27])$ \\
\hline \multicolumn{6}{|c|}{ Emotion Attribution Task } \\
\hline Sadness & 7 & 7 & 7 & 7 & 7 \\
\hline Embarrassment & 6 & 7 & 8 & 7 & 8 \\
\hline Envy & 2 & 2 & 0 & 2 & 1 \\
\hline Anger & 7 & 5 & 4 & 6 & 7 \\
\hline Fearfulness & 8 & 7 & 7 & 8 & 8 \\
\hline Disgust & 1 & 1 & 2 & 2 & 2 \\
\hline Happiness & 9 & 8 & 10 & 9 & 10 \\
\hline Advanced Theory of Mind Task & 8 & 10 & 9 & 12 & $\geq 12$ \\
\hline \multicolumn{6}{|c|}{ Basic Empathy Scale } \\
\hline BES-Affective & 31 & 31 & 30 & 32 & - \\
\hline BES-Cognitive & 23 & 26 & 20 & 27 & - \\
\hline
\end{tabular}

Table 2: Results of tests administered during the pre-intervention phase were compared with normative data (global cut-off).

The Wilcoxon signed-rank test was used to evaluate the changes in social cognition measures as a result of the treatment. Our results showed that the four young adults improved their performance on cognitive measures of social cognition, in particular on the BES- cognitive subscale $(\mathrm{Z}=-1.84 ; \mathrm{P}=0.044)$ and in the Advanced Theory of Mind Task $(\mathrm{Z}=-1.826 ; \mathrm{P}=0.048)$. No improvements in the affective component of empathy were found (Table 3 ).

\begin{tabular}{|c|c|c|c|c|}
\hline & PRE & POST & $\mathbf{z}$ & $\mathbf{P}$ \\
\hline Eyes Task & $20(7.25)$ & $19(8.08)$ & 0.184 & 0.854 \\
\hline \multicolumn{5}{|c|}{ Emotion Attribution Task } \\
\hline Sadness & $7.25(1.25)$ & $7(1.41)$ & 0.378 & 0.705 \\
\hline Embarrassment & $4(4.08)$ & $3.25(4.03)$ & 0.184 & 0.854 \\
\hline Envy & $0.5(1)$ & $0.75(1.5)$ & -1 & 0.317 \\
\hline Anger & $5.5(3)$ & $6.5(2.38)$ & -1.633 & 0.102 \\
\hline Fearfulness & $8.25(0.95)$ & $8.5(1)$ & -0.577 & 0.564 \\
\hline Disgust & $1.75(1.25)$ & $2.5(1)$ & -1.732 & 0.083 \\
\hline Happiness & $9.25(0.95)$ & $10(0)$ & -1.342 & 0.18 \\
\hline Advanced Theory of Mind Task & $7(4.69)$ & $10.5(3.31)$ & -1.841 & 0.044 \\
\hline \multicolumn{5}{|c|}{ Basic Empathy Scale } \\
\hline BES-Affective & $31(0.81)$ & $35(4.65)$ & -1.289 & 0.197 \\
\hline
\end{tabular}




\section{BES-Cognitive}

$24(3.16)$

$32(3.55)$

$-1.826$

0.048

Table 3: Comparison between pre and post treatment phases.

\section{Discussion}

This pilot study is among the first to investigate the use of an intervention based on the interaction of individuals with ASD to enhance social skills and social cognition. During the intervention sessions, the young adults had an important goal: to create software aimed at improving the emotional competence and social interaction in children with their same syndrome. All four young adults are experts in technology and learn in autodidactic way, but they show social and emotional deficits. Kumschick and collaborators highlighted the importance of emotional competence as an ability that is teachable and learnable [28]. We believe that the use of software that focuses on social and emotional relationships, such as "The Transporters", could help children with ASD improve their social competence [29]. "The Transporters" is an animated series designed to enhance emotion comprehension in children with ASD. In Golan et al.s study, 20 children with ASD (aged 4-7) watched "The Transporters" every day for four weeks. The authors concluded that using "The Transporters" significantly improved emotion recognition in children with ASD [29]. The innovative aspect of our study was that young adults with autism created the software for use by children with autism. Only individuals that present social difficulties are able to recreate the daily problems that characterise their life and, at same time, also reflect on how to overcome these problems and suggest solutions to others.

The young adults were tested before and after the intervention on emotion recognition and empathic ability. Our results showed an qualitative and quantitative improvement of their cognitive empathic abilities, as witnessed by parents and scores obtained of the test post training (i.e., the ability to understand the emotional state of other people and the ability to make inferences about emotions) [5,8-10,30]. One set of parents noticed a change in their son's behaviour and in his ability to interact with others; the young adults were more willing to discuss emotions. No improvements in the affective component of empathy were found, however. We support the idea that the cognitive aspect of empathy is a prerequisite for intact development of affective empathy processing. Thus, we believe that there is need for more intense and prolonged treatment to improve the affective component of empathic ability. Our young adults showed an enhancement of emotional competence.

Recent studies showed that children with a high level of emotional competence are more successful at interacting with their peer group $[28,31,32]$. On the contrary, children with a low level of emotional knowledge are often rejected by peers and can develop social anxiety and behaviour problems $[28,33]$.

Kamps and collaborators reported that social interventions that target well-defined skills and have a behavioural teaching component could improve social and communicative skills [15]. The results of their study showed that most children were immediately responsive to the PMI and learned to communicate with their peers during treatment sessions. In addition, children who received the PMI showed significantly more growth in initiations with peers within the nontreatment social probe sessions that more closely resembled a free play with toys and games' social setting, as well as during generalisation settings (recess, centres, lunch), than did the comparison group.
Overall, the current investigation provides preliminary evidence for the feasibility of involving individuals with ASD in creating intervention programmes directed at children with autism. This intervention has permitted to young adults to be active participants during the treatment. This is a very important aspect for young adults with ASD transitioning into adulthood. This is a pilot study and our sample was very small; as a matter of fact, this is an important limitation to our study. However, we believe in the importance of our results in the rehabilitative field. In fact, the process of development of software as a therapeutic context represents an innovative approach. The next step will be to evaluate the efficiency of software created by young adults in children with ASD on social and emotional abilities. In addition, a six-month follow-up will be also conducted on social cognition measures to examine the long term effect on the social and emotional functioning of our four young adults.

\section{References}

1. Abrahams BS, Geschwind DH (2008) Advances in autism genetics: on the threshold of a new neurobiology. Nat Rev Genet 9: 341-355.

2. Franco F, Itakura S, Pomorska K, Abramowski A, Nikaido K, et al. (2014) Can children with autism read emotions from the eyes? The eyes test revisited. Res Dev Disabil 35: 1015-1026.

3. American Psychiatric Association (2013) Diagnostic and statistical manual of mental disorders. 5th ed. Washington.

4. Lai MC, Lombardo MV, Baron-Cohen S (2014) Autism. Lancet 383: 896-910.

5. Mazza M, Pino MC, Mariano M, Tempesta D, Ferrara M, et al. (2014) Affective and cognitive empathy in adolescents with autism spectrum disorder. Front Hum Neurosci 8: 791.

6. Mazza M, Lucci G, Pacitti F, Pino MC, Mariano M, et al. (2010) Could schizophrenic subjects improve their social cognition abilities only with observation and imitation of social situations? Neuropsychol Rehabil 20: 675-703.

7. Premack D, Woodruff G (1978) Does the chimpanzee have a theory of mind? Behav Brain Sci 1: 515-526.

8. Shamay-Tsoory SG, Harari H, Aharon-Peretz J, Levkovitz Y (2010) The role of the orbitofrontal cortex in affective theory of mind deficits in criminal offenders with psychopathic tendencies. Cortex 46: 668-677.

9. Sebastian CL, Fontaine NM, Bird G, Blakemore SJ, Brito SA, et al. (2012) Neural processing associated with cognitive and affective Theory of Mind in adolescents and adults. Soc Cogn Affect Neurosci 7: 53-63.

10. Mazza M, Tempesta D, Pino MC, Nigri A, Catalucci A, et al. (2015) Neural activity related to cognitive and emotional empathy in posttraumatic stress disorder. Behav Brain Res 282: 37-45.

11. Vetter NC, Leipold K, Kliegel M, Phillips LH, Altgassen M (2013) Ongoing development of social cognition in adolescence. Child Neuropsychol 19: 615-629.

12. Volkmar F, Carter A, Grossman J, Klin A (1997) Social development in autism. Handbook of autism and pervasive developmental disorders. New York.

13. Goldstein H, Schneider N, Thiemann K (2007) Peer-mediated social communication intervention: When clinical expertise informs treatment development and evaluation. Top Lang Disord 27: 182-199.

14. Jones CD1, Schwartz IS (2009) When asking questions is not enough: an observational study of social communication differences in high functioning children with autism. J Autism Dev Disord 39: 432-443. 
Citation: Mazza M, Pino MC, Mariano M, Valenti M (2016) An Innovative Approach to Development of Social Abilities in Individuals with Autism: A Pilot Study. Autism Open Access 6: 163. doi:10.4172/2165-7890.1000163

Page 5 of 5

15. Kamps D, Thiemann-Bourque K, Heitzman-Powell L, Schwartz I Rosenberg N, et al. (2015) A comprehensive peer network intervention to improve social communication of children with autism spectrum disorders: a randomized trial in kindergarten and first grade. J Autism Dev Disord 45: 1809-24.

16. Bellini S, Peters J, Benner L, Hopf A (2007) Meta-Analysis of schoolbased social skills interventions for children with autism spectrum disorders. Remedial Spec Educ 28: 153-162.

17. Reichow B, Volkmar FR (2010) Social skills interventions for individuals with autism: evaluation for evidence-based practices within a best evidence synthesis framework. J Autism Dev Disord 40: 149-166.

18. Taylor JL, Seltzer MM (2010) Changes in the autism behavioral phenotype during the transition to adulthood. J Autism Dev Disord 40: 1431-1446.

19. Pilling S, Baron-Cohen S, Megnin-Viggars O, Lee R, Taylor C; Guideline Development Group (2012) Recognition, referral, diagnosis, and management of adults with autism: summary of NICE guidance. BM] 344: e4082.

20. Lord C, Rutter M, Di Lavore PC, Risi S, Gotham K, et al. (2012) Autism Diagnostic Observation Schedule (ADOS-2), Los Angeles.

21. Wechsler D (1997) Adult Intelligence Scale, Wechsler.

22. Blair RJ, Cipolotti L (2000) Impaired social response reversal. A case of 'acquired sociopathy'. Brain 123: 1122-1141.

23. Happé FG (1994) An advanced test of theory of mind: understanding of story characters' thoughts and feelings by able autistic, mentally handicapped, and normal children and adults. J Autism Dev Disord 24 129-154.

24. Jolliffe D, Farrington DP (2006) Development and validation of the Basic Empathy Scale. J Adolesc 29: 589-611.

25. Albiero P, Matricardi G, Speltri D, Toso D (2009) The assessment of empathy in adolescence: A contribution to the Italian validation of the "Basic Empathy Scale". J Adolesc 32: 393-408.
26. Carré A, Stefaniak N, D'Ambrosio F, Bensalah L, Besche-Richard C (2013) The Basic Empathy Scale in adults (BES-A): factor structure of a revised form. Psychol Assess 25: 679-691.

27. Baron-Cohen S, Wheelwright S, Hill J, Raste Y, Plumb I (2001) The "Reading the Mind in the Eyes" Test revised version: a study with normal adults, and adults with Asperger syndrome or high-functioning autism. J Child Psychol Psychiatry 42: 241-251.

28. Kumschick IR, Beck L, Eid M, Witte G, Klann-Delius G, et al. (2014) READING and FEELING: the effects of a literature-based intervention designed to increase emotional competence in second and third grader. Front. Psychol 5: 1448

29. Golan O, Ashwin E, Granader Y, McClintock S, Day K, et al. (2010) Enhancing emotion recognition in children with autism spectrum conditions: an intervention using animated vehicles with real emotional faces. J Autism Dev Disord 40: 269-279.

30. Shamay-Tsoory SG1 (2011) The neural bases for empathy. Neuroscientist 17: $18-24$

31. Schultz D1, Izard CE, Ackerman BP, Youngstrom EA (2001) Emotion knowledge in economically disadvantaged children: self-regulatory antecedents and relations to social difficulties and withdrawal. Dev Psychopathol 13: 53-67.

32. Smith M (2001) Social and emotional competencies: contributions to young African-American children's peer acceptance. Early Educ Dev 12: 49-72.

33. Speltz ML, McClellan J, DeKlyen M, Jones K (1999) Preschool boys with oppositional defiant disorder: clinical presentation and diagnostic change. J Am Acad Child Adolesc Psychiatry 38: 838-845. 